\title{
Conversion Electrons in the SDC
}

\author{
A. B. Wicklund
}

We summarize a preliminary analysis of the rates for conversion electrons in the SDC detector, relative to other interesting sources of prompt electrons. We have used Papageno V3.30, and other available NLO calculations to estimate inclusive rates in the central region ( $\eta$ less than 2.0), and we have cross checked these using CDF data at 1.8 TeV. We have considered three sources of "isolated" electrons, namely

(1) Inclusive $W / Z$ production.

(2) Top quark ( $M t=140)$.

(3) QCD prompt photon production, followed by conversion in 10\% X0. This value approximates the inner silicon detector at SDC. Additional conversions will occur in the outer tracking chamber, but the trigger and track reconstruction efficiency will be lower.

We have also considered "nonisolated" leptons coming from

(4) Inclusive Bottom production

(5) Photon conversions resulting from $\pi^{0}, \eta$ production in jets.

(6) High Pt hadrons faking electrons.

The efficiency for triggering and reconstructing nonisolated electrons is detector dependent, but we can get an idea of the relative rates for these "electrons" from the CDF data. While the $\mathrm{SDC}$ cross sections are typically $\mathrm{A} 10$ higher, and harder in $\mathrm{Pt}$, the relative rates for the parton level processes,

(a) $W+$ jet(s)

(b) QCD Photon + jets

(c) $b+\operatorname{Jet}(s)$

(d) $\pi^{0} \rightarrow \gamma$, or fake hadron

all scale rather similarly as functions of $\mathrm{Pt}$ or $\mathrm{Xt}$.

For process (a), the full NLO calculation (Arnold,Kauffman, Reno) agrees with the CDF $\mathrm{Pt}(\mathrm{W}, \mathrm{Z})$ distributions at $1.8 \mathrm{TeV}$; lowest order Papageno $(W+1$ jet) gives an adequate 
description of these data, provided an overall $\mathrm{K}=2.2$ factor is applied to Papageno. For process (b), we have NLO predictions by Aurenche at 1.8 and $40 \mathrm{TeV}$, the forner in fair agreement with CDF data; the NLO predictions agree with lowest order Papageno $(\gamma+1$ jet), using a $K=3$ factor. For process (c), the NLO rates predicted by Nason,Ellis, Dawson require a $K=3$ factor, compared with lowest order Papageno. Figure (1) shows the inclusive electron rates at CDF for $\eta \leq 0.7,4.1 \mathrm{pb}-1$. The electron isolation cuts give an efficiency of $40-50 \%$ on the low $\mathrm{Pt}$ continuum, which includes bottom electrons (60\%), non-identified conversions (18\%), and hadron fakes (22\%). The $\mathrm{W} / \mathrm{Z}$ jacobean peak is evident. The total rate for identified conversions, which are removed from fig. 1 , is approximately $28 \%$ of the low $\mathrm{Pt}$ continuum in fig. 1. Of the found conversions, we estimate that $30-35 \%$ come from QCD prompt photon production, based on their isolation properties. The remaining found conversions are presumably due to $\pi^{0}, \eta$ decays in jets. The nonidentified conversions (18\% of the low $\mathrm{Pt}$ continuum) are conversion pairs in which the partner positron has low momentum, so that the track reconstruction efficiency is low.

It may seem odd that such a large fraction of found conversions come from QCD prompt photon production, rather than $\pi^{0}, \eta$ decay. The reason is trigger bias. The CDF electron trigger requires a high $\mathrm{Pt}$ track in association with a high Et calorimeter deposition, and of course the higher level trigger and offline selection code tightens that requirement. Thus, while a symmetric $\pi^{0}$ decay can satisfy an Et requirement (eg,a "photon trigger"), only asymmetric $\pi^{0}$ decays can give a conversion that satisfies the prompt electron trigger. Figure 2 shows the energy and momentum in a cone of radius 0.7 , around prompt electron candidates. The histogram is for identified conversions, while the shaded plot is for other electrons (b-decays, etc), normalized on the right-side of the plot. The quantity plotted is the sum of calorimeter Et and the sum of track momenta, added in quadrature (excluding the electron or conversion pair). The peak at small isolation energy comes from QCD prompt photon production. Figure (3) shows the same distribution for the conversion sample after subtracting the "background" from nonisolated conversions (we use the shaded curve in fig.(2), to describe the background shape.). The histogram shows the same distribution for electrons from $W$ decay, normalized in the first bin. The isolation distributions are evidently quite similar for the "isolated conversions" and the $W$ electrons, provided we exclude the conversion "partner" from the Et,Pt sum.

Figure 4 shows the predicted electron spectra at $1.8 \mathrm{TeV}$, averaged over $(\eta \leq 2)$, from W decay and QCD prompt photons (we use Bethe Heitler to describe the photon conversion to electron pairs, and integrate the total electron rate with no cuts on tagging efficiency.) The solid curve for prompt photons corresponds to $10 \% \mathrm{X0}$. The dashed curve is an interpolation of the rate for isolated conversion electrons seen in CDF (the background-subtracted peak in fig.2). We have normalized the observed conversion rate to the $W$ electron peak. The dashed curve is consistent with the solid curve prediction for prompt photons, taking into account the $4.6 \% \mathrm{XO}$ equivalent radiator inside the CDE central tracker. Figure 5 shows the same rates for the SDC. Both at CDF and SDC, the prompt photon curve crosses the $\mathrm{W} \rightarrow e^{+-}$ curve at around $25 \mathrm{GeV} / \mathrm{c} \mathrm{Pt}$. Also shown in fig. 5 is the lowest order $\mathrm{T}$ Tbar $(\mathrm{Mt}=140)$ rate 
from Papageno. We note that the photon predictions are NLO from Aurenche, as described above; the W curve at 1.8 is from the Arnold et al NLO prediction, which agrees with CDF data, and the $W$ curve at $40 \mathrm{TeV}$ is Papageno with a $\mathrm{K}$ factor=2.2. (We have included the Papageno $\mathrm{W}+0$ jet rates with $K=1$.) We enphasize that the $\mathrm{W}$ rates do not include $\mathrm{Z} \rightarrow e+e-;$ to add these, multiply the $W$ rates by 1.2 .

Aside from the overall $\mathrm{X} 10$ rate increase at the SDC, the $\mathrm{W} \rightarrow e+-$ curves are similar at the two energies, simply because the electron $\mathrm{Pt}$ spectrum is doninated by the jacobean peak in the $\mathrm{W} \rightarrow e v$ decay; this peak is smeared more at the higher energy, due to the harder intrinsic $\mathrm{Pt}(\mathrm{W})$ spectrum. With a "reasonable" high luminosity trigger, Et $>50$, integrated over +-2 units of $\eta$, we expect around $2 \mathrm{~Hz}$ at $10^{* *} 33$ from $W$ and Top electrons, with $40 \%$ conversion background. This may be a reasonable choice for an inclusive single electron trigger, which has been used successfully for virtually all of the heavy flavor and electroweak studies carried out at CDF (CDF used a $12 \mathrm{GeV}$ Et threshold.)

To summarize, the rates for isolated electrons from $Q C D$ prompt photons, relative to $\mathrm{W} \rightarrow \mathrm{e}+-$, Top $\rightarrow$ ef- etc., are expected to be tolerable with $10 \%$ X0 effective radiator in the silicon detector. In particular, the isolated conversions, illustrated in figs. 4,5, should have similar isolation properties (except for the presence of a partner positron) as the electrons from $W$ and Top sources; they should give about half the $W \rightarrow e+-$ rate for $E t \geq 50 \mathrm{GeV}$. From kinematics, we would expect the conversion finding efficiency to be high ( $80 \%$ ), but this needs more detailed simulation. If the conversion identification is well understood, these isolated conversions can be used as a control sample in studies of lepton plus multijet funal states (as at CDF.) The isolated conversions are only part of a low $P t$ continuum, which will include nonisolated conversions, bottom/charm decays, and hadron fakes. If we extrapolate these rates from CDF, assuming the same efficiencies for nonisolated electrons, we predict the following mix for the low $\mathrm{Pt}(\mathrm{Pt} \leq 20)$ continuum:

$\begin{array}{llll} & & \text { CDF } & \text { SDC } \\ \text { (1) b,c,fakes } & 64 \% & 47 \% \\ \text { (2) Unseen Conversions } & 14 \% & 21 \% \\ \text { (3) Seen Conversions } & 22 \% & 32 \% \\ & \text { Total } & 100 \% & 100 \% \\ \text { (4) Isolated Conversions } & 11 \% & 16 \%\end{array}$

The precise mix depends on isolation cuts. We are assuming that the main sources (b production and $\pi^{0}$ decays) scale with $X t$ as the prompt photon rate. Taking into account the softer $\mathrm{Pt}$ spectrum for nonisolated electrons, one would expect this low Pt continuum to extend to the $E t \geq 50$ regions in figs. 4,5, with rates comparable to those from isolated conversions.

Figure (6) shows an estimate for the b+bbar inclusive cross section at $40 \mathrm{TeV}$, averaged over $\eta<1.5$, based on the Nason, Ellis, Dawson calculation (computation courtesy of $R$. Meng, priv. comm.). Also shown is the corresponding $\mathrm{Pt}$ spectrum for electrons from these $b$ decays, assuming an average $12 \% \mathrm{~b} \rightarrow \mathrm{e}$ B.R.; there is about an order of magnitude reduction 
due to the kinematics of $b \rightarrow e$ decay (eg $\operatorname{Pt}(e+-) \ll P t(b)$ ), in addition to the B.R. Using an approxinate (CDF based) sinnuation of the effects of a Had/EM cut, where we require $\mathrm{Had} / \mathrm{En}<0.04$ in a $0.2 \times 0.2 \mathrm{Had} / \mathrm{EM}$ region, we get the lower curve for "nonisolated" electrons from b-decay. There will be additional acceptance losses associated with overlap of $\pi^{0}$ showers with the electron, but these are not included in this simulation. The acceptance losses associated with a nominal Had/EM cut become quite severe for $\mathrm{Pt}(\mathrm{e}+-)>20 \mathrm{GeV} / \mathrm{c}$, due to the hadronic fragments accompanying the electron (note that the B-meson mass and the transverse size of the hadron jet are fixed.) The actual inclusive b-quark cross section at SDC may be significantly higher than the NSD calculation (CDF observes $2.4 \mathrm{X}$ the NSD rate). In general, as expected the b-rates are comparable to those for conversion electrons, depending on the actual enhancement over QCD.

\section{Figure Captions}

1. Inclusive electron spectrum from $\mathrm{CDF}$; dahsed curve shows expected charm contribution, solid shows bottom+charm. Crosses denote electron rates with $\mathrm{W}, \mathrm{Z}$ electrons subtracted.

2. Isolation distribution for conversion electrons in CDF (total histogram), and for prompt electrons (shaded region.)

3. Isolation distribution for conversion electrons in CDF, using prompt electrons to provide background subtraction shape (points), and isolation distribution for $W$ electrons (histogram.)

4. Predicted electron rates at $\mathrm{CDF}$ from $\mathrm{W}$ decay and from prompt photon conversions with $10 \%$ X0. Dashed curve shows observed CDF rate for $4.6 \%$ X0.

5. Same as (4) for $40 \mathrm{TeV}$, including $\operatorname{Top}(M=140)$ contribution.

6. Inclusive rates for bottom quark ( $b$ plus bbar) production in $\eta \leq 1.5$, and for prompt e+- from b-quark decay with no efficiency cuts (top curve) and with expected Had/Em cut (bottom curve.) Electron rates include $12 \%$ semileptonic B.R.

This work supported by the U. S. Department of Energy, Division of High Energy Physics, Contract W-31-109-ENG-38.

\section{DISCLAIMER}

This report was prepared as an account of work sponsored by an agency of the United States Government. Neither the United States Government nor any agency thereof, nor any of their employees, makes any warranty, express or implied, or assumes any legal liability or responsibility for the accuracy, completeness, or usefulness of any information, apparatus, product, or process disclosed, or represents that its use would not infringe privately owned rights. Reference herein to any specific commercia! product, process, or service by trade name, trademark, manufacturer, or otherwise does not necessarily constitute or imply its endorsement, recommendation, or favoring by the United States Government or any agency thereof. The views and opinions of authors expressed herein do not necessarily state or reflect those of the United States Goversment or any agency thereof. 
Inclusive Electron Spectrum

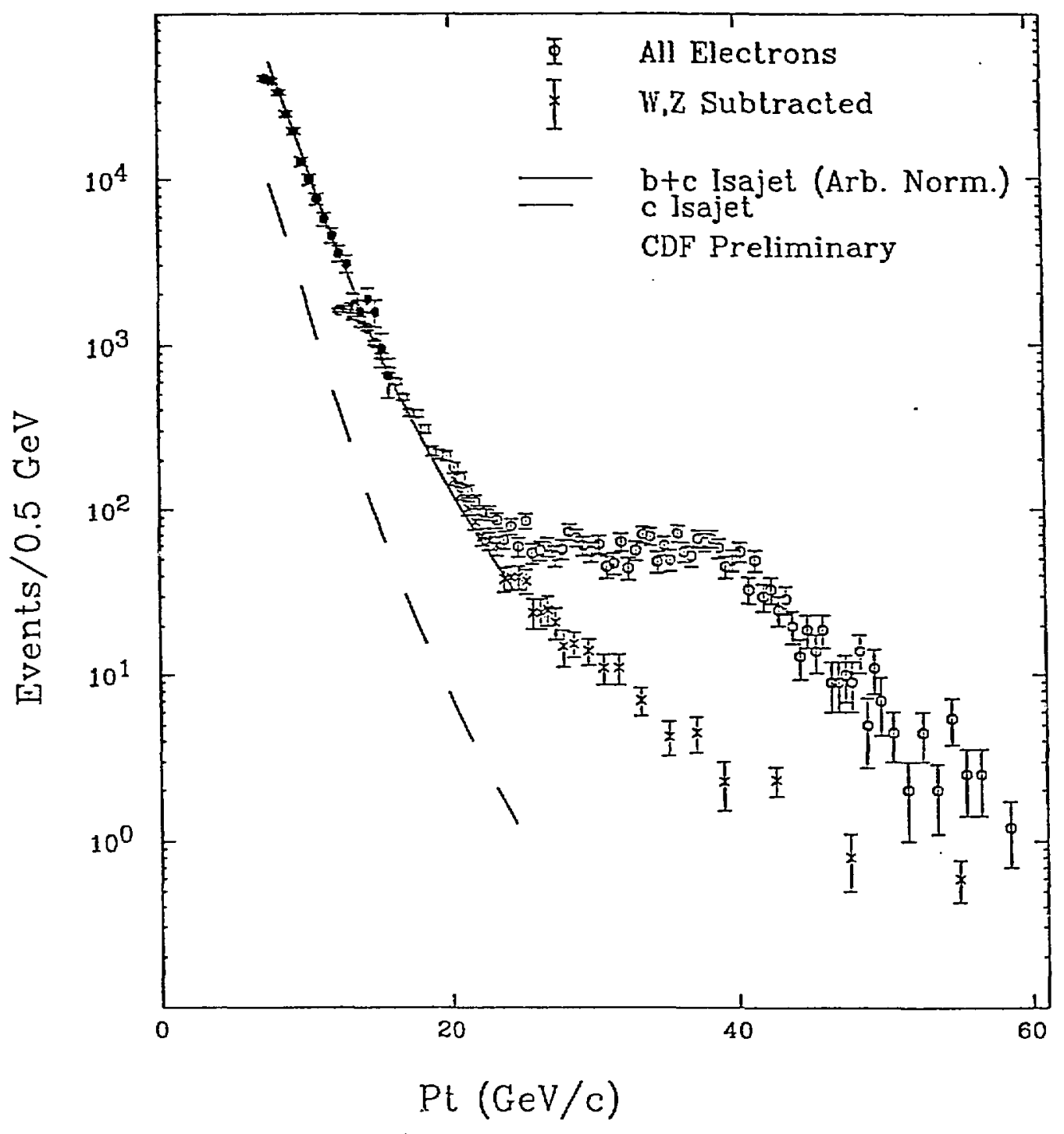

(Fi3. I) 


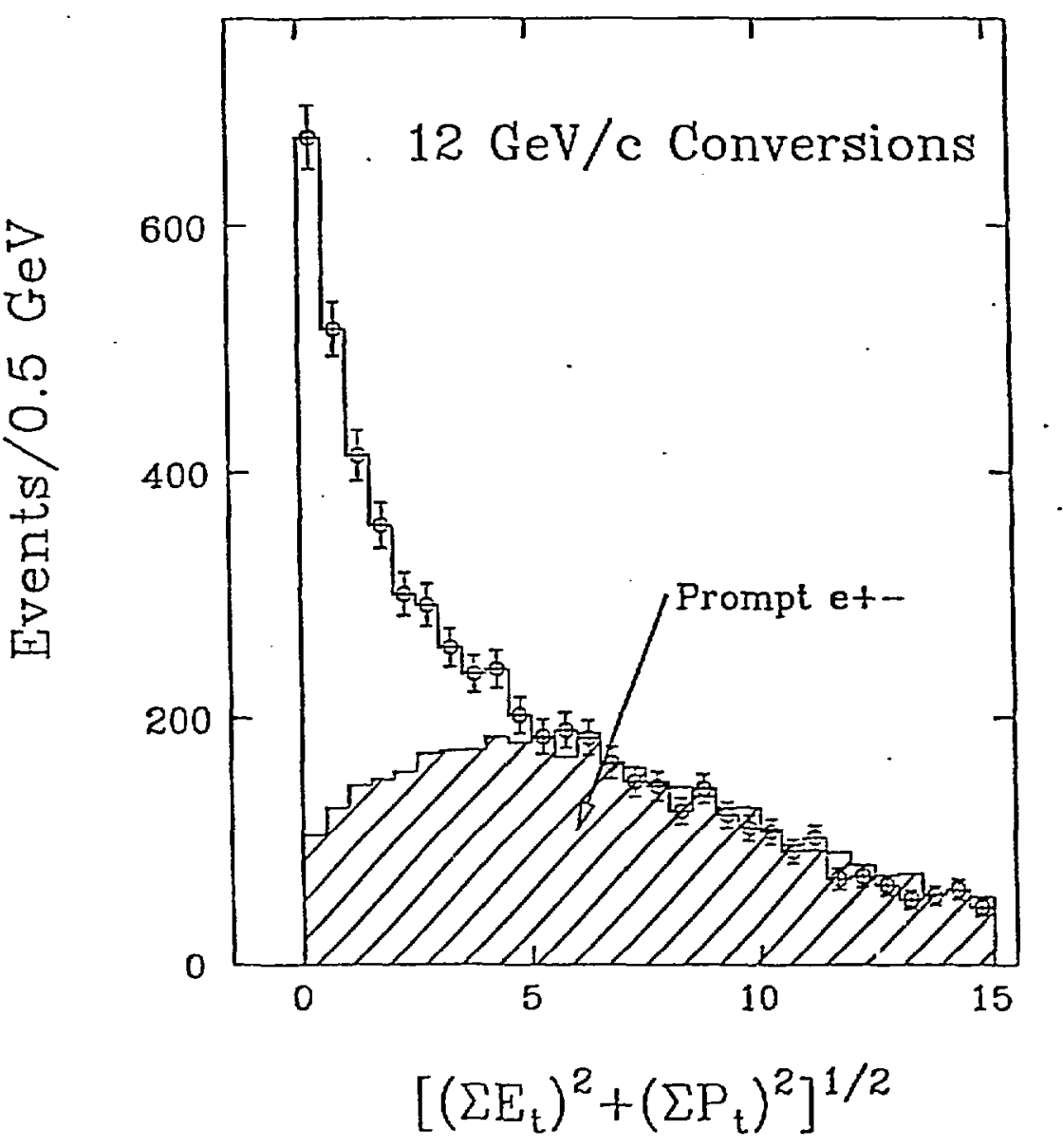

Fig. (2) 


\section{Events/0.5 GeV}

$\frac{\pi}{01}$
$\frac{10}{2}$

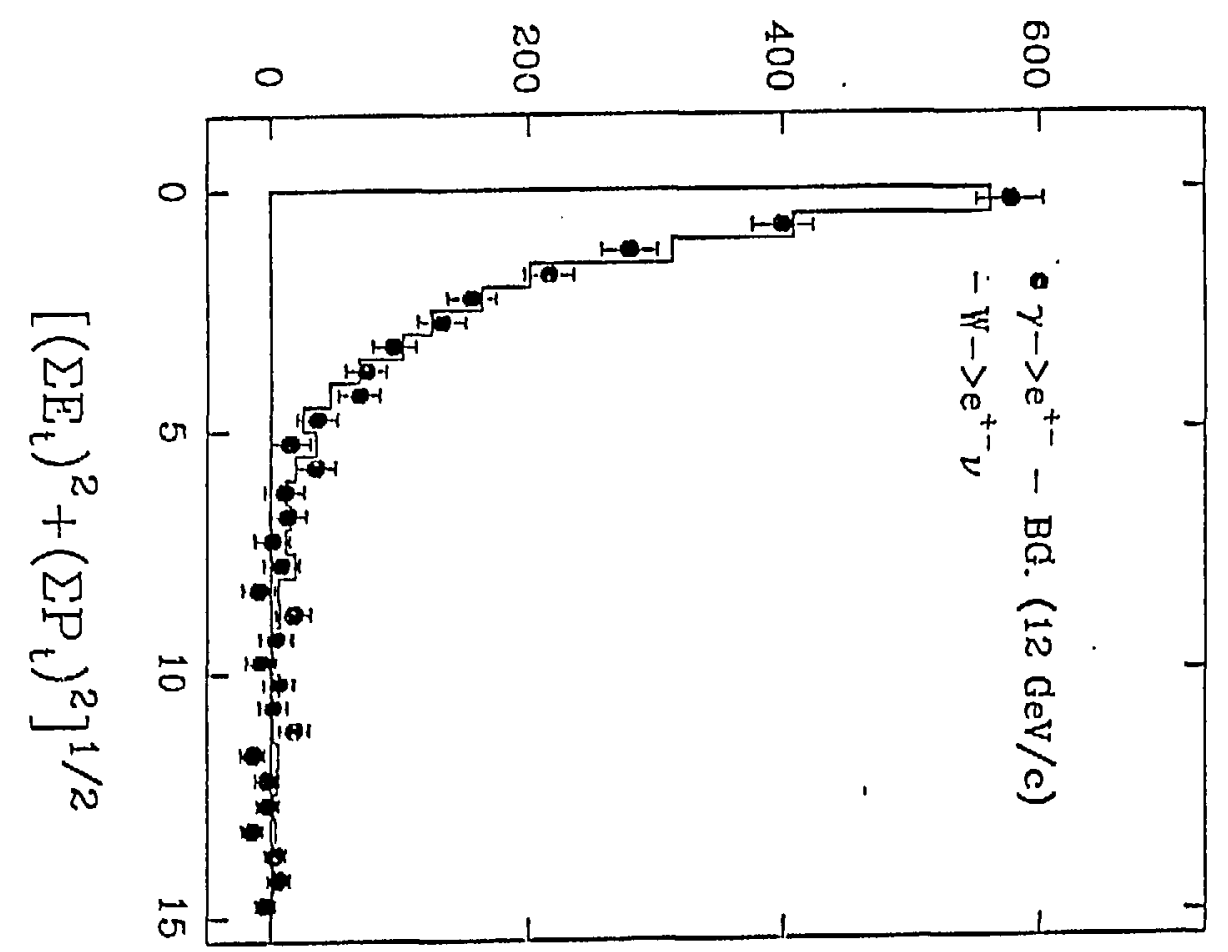




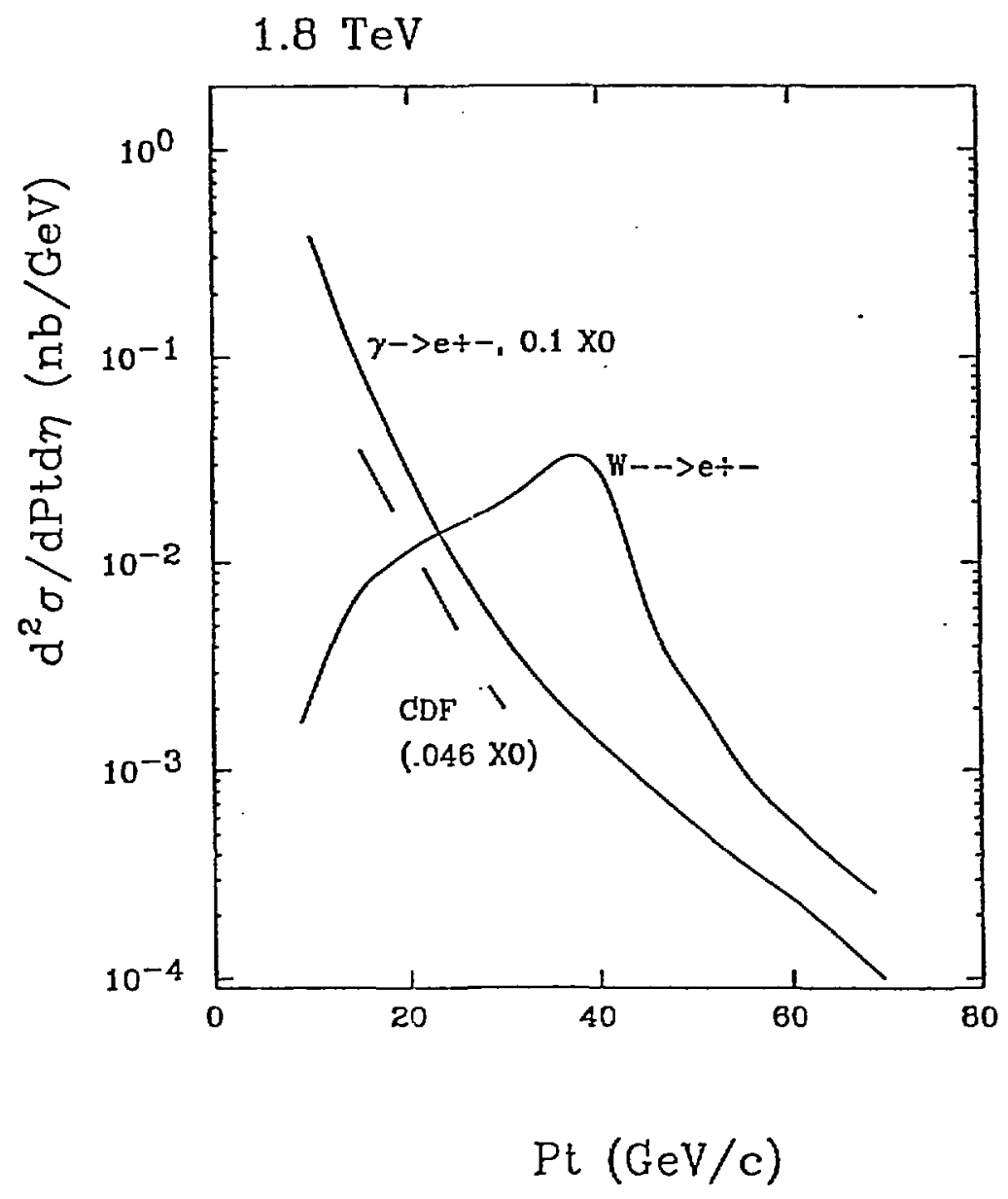

(Fig. 4) 


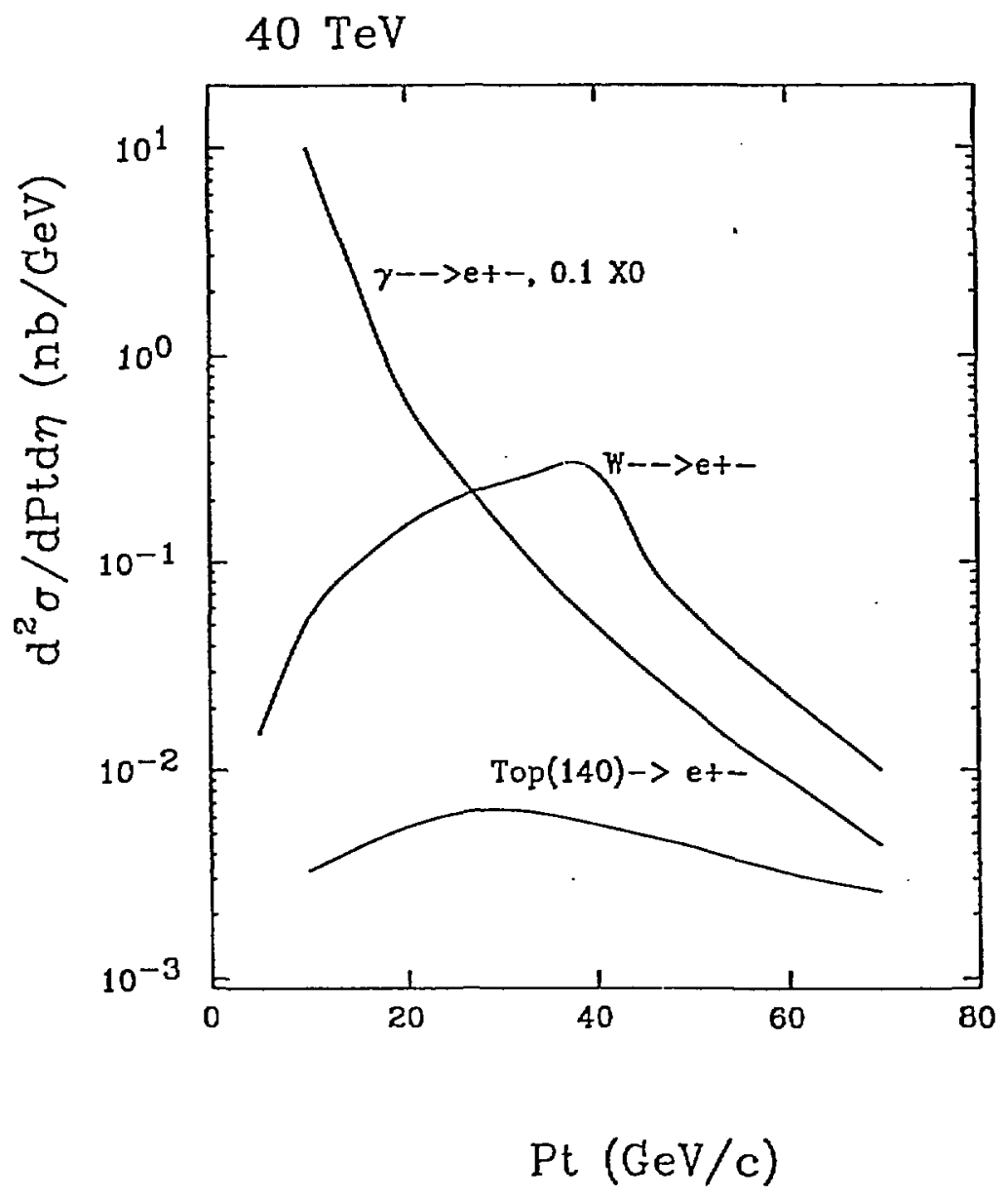

(Fig. 5) 


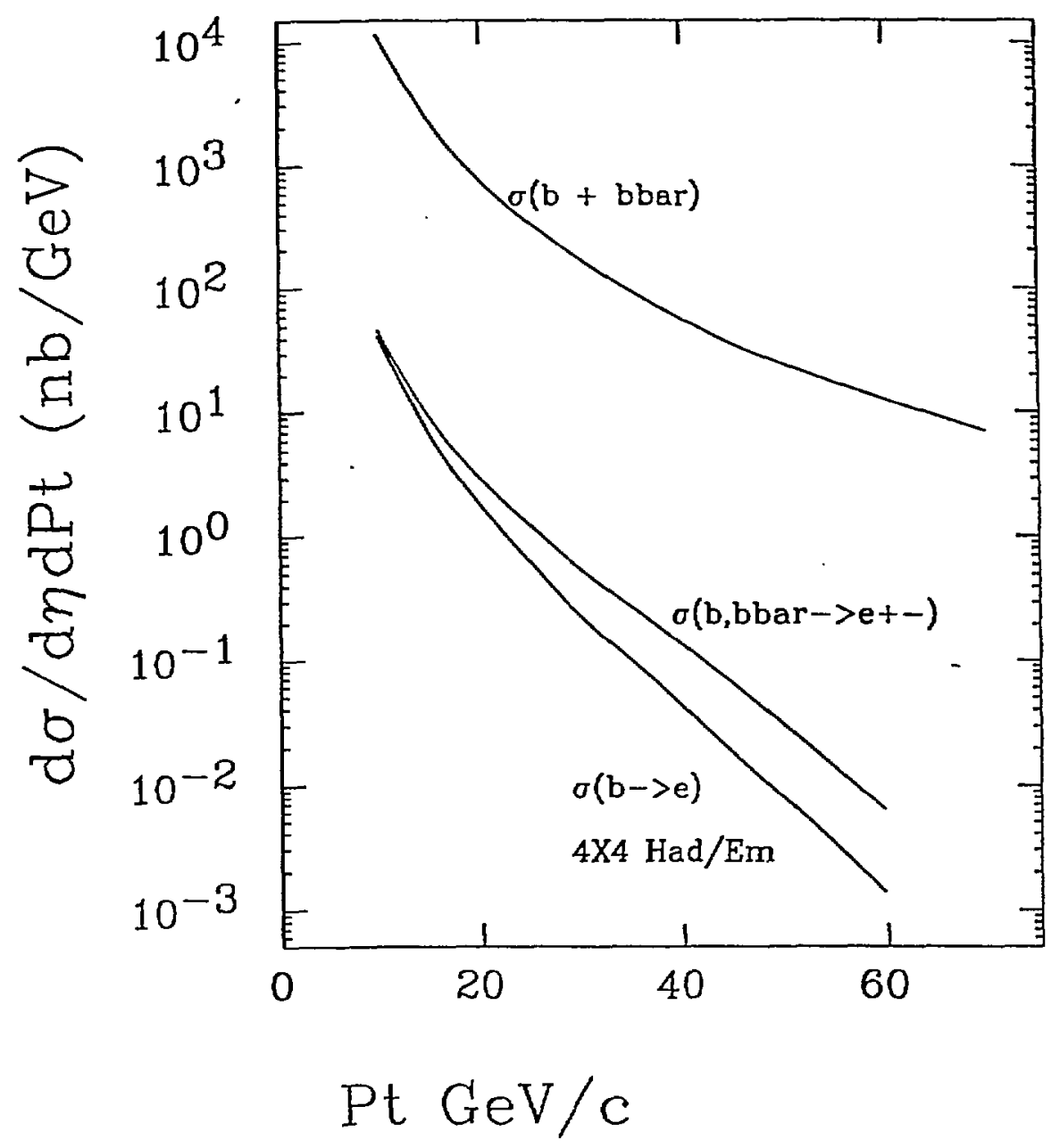

$F_{i g}(6)$ 